\title{
MASSIVE LOCALLY EXTENSIVE PROSTATE CANCER
}

\author{
THOMAS A. ROZANSKI, M.D. \\ GARY J. FAERBER, M.D.
}

From the Department of Surgery, Section of Urology, University of Michigan, Ann Arbor, Michigan

\begin{abstract}
A massive, locally extensive adenocarcinoma of the prostate $(2,246 \mathrm{ccm})$ presenting as constipation and a palpable abdominal mass, was treated with androgen deprivation resulting in a 94 percent reduction in tumor size, and relief of symptoms. Serum prostate-specific antigen was reduced from 4,029 to $0.4 \mathrm{ng} / \mathrm{mL}$, after hormonal therapy was combined with radiation treatments for local disease control.
\end{abstract}

A sixty-seven-year-old man presented to our urology clinic in April 1991 with a two-month history of worsening constipation, obstructive and irritative voiding symptoms, and pelvic pain. An abdominal mass was palpable up to the umbilicus. Digital rectal examination revealed a large, hard mass consistent with prostatic carcinoma. Post void residual was unremarkable, mean urinary flow rate was $5.4 \mathrm{cc} / \mathrm{sec}$, and serum prostate-specific antigen (PSA) was 4,028.9 ng/mL. Prostate biopsy specimen showed adenocarcinoma, Gleason score 7. Abdominal and pelvic computed tomography (CT) scan revealed a massive pelvic and abdominal mass measuring $20 \times 18 \times 12 \mathrm{~cm}$, displacing the bladder anteriorly and the rectum posteriorly (Fig. 1). Bilateral hydronephrosis and hydroureter to the level of the mass was evident, as was periaortic and pericaval lymphadenopathy. A chest x-ray film and nuclear bone scan findings were negative for tumor, and the patient's disease was staged as D2 because of the retroperitoneal adenopathy.

Androgen deprivation was initiated using leuprolide, $7.5 \mathrm{mg}$ intramuscularly every four weeks, and flutamide, $250 \mathrm{mg}$ orally three times daily. Follow-up CT scan in November 1991 revealed a marked decrease in tumor mass to $5 \times 7 \times 7 \mathrm{~cm}$ (94.3\% volume reduction) (Fig. 2). With shrinkage of the tumor mass, the hydronephrosis was resolved, and seminal vesicle involvement and pelvic lymphadenopathy were demonstrated. Serum PSA values gradually decreased to 499.5

Submitted: August 7, 1993, accepted: September 10, 1993

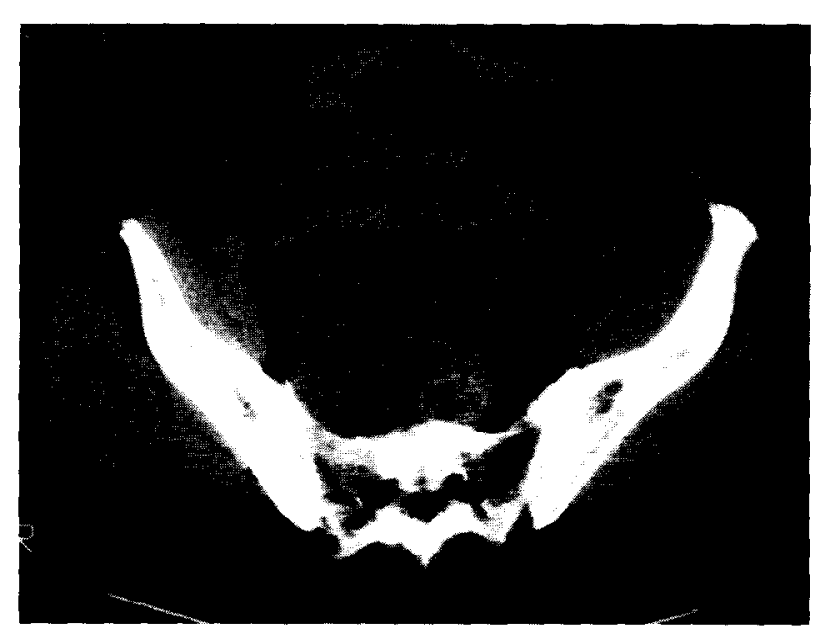

FIGURE 1. CT scan demonstrating large tumor mass with displacement of bladder and rectum.

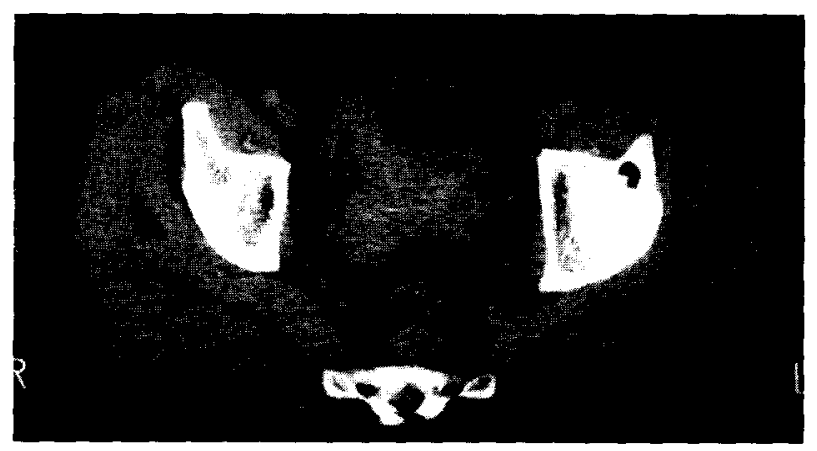

FIGURE 2. CT scan after six months of leuprolide injections, showing marked reduction of tumor mass.

$\mathrm{ng} / \mathrm{mL}$ after eight months of androgen deprivation. With the significant response to hormonal therapy, external beam radiation therapy was 
instituted for local disease control with $6,000 \mathrm{R}$ delivered over a seven-week period beginning in December 1991.

Serum PSA continued to decline, reaching 43.7 $\mathrm{ng} / \mathrm{mL}$ one year after radiation therapy. The patient became and continues to be asymptomatic, without constipation, voiding complaints, or pelvic pain. A repeat bone scan in January 1993 was normal, and his most recent PSA value in July 1993, twenty-seven months after initiating androgen deprivation and eighteen months since completing radiation therapy, was $0.4 \mathrm{ng} / \mathrm{mL}$, a 99.99 percent reduction.

\section{COMMENT}

This case report describes an interesting and unusual presentation of prostate cancer, and an equally unusual response to therapy. Rarely does adenocarcinoma of the prostate present with constipation and a palpable abdominal mass. It is also unusual to have such massive tumor burden and elevated PSA without bony metastases.

Several authors have reported using gonadotropin releasing hormone agonists in an attempt to downstage locally extensive prostatic carcinoma prior to radical prostatectomy. Preoperative androgen suppression results in marked lowering of serum PSA values, averaging a 94 to 98.5 percent reduction. ${ }^{1-3}$ However, this laboratory response does not translate into a change in the pathologic stage, DNA ploidy, or Gleason score, when initial biopsy pretreatment data are compared with findings at prostatectomy. ${ }^{1-3}$ Preoperative hormonal therapy does reduce prostate volume. Size reduction varies, but has been reported to be between 25 and 55 percent as determined by transrectal ultrasound, when prostate cancer is treated with various methods of androgen suppression for two to five months. ${ }^{1,2}$ Androgen deprivation appears to downsize, but not downstage prostate cancer. A recent study from Norway, in which benign prostatic hyperplasia was treated with leuprolide, reported an average decrease of 34.5 percent in prostate volume after twenty-four weeks of treatment. ${ }^{+}$The variability in prostate volume reduction can be related to length of treatment, method of hormonal manipulation, the fact that androgen suppression causes prostate cell atrophy and not cell death, and the accepted concept of a heterogeneous population of prostatic androgen sensitive and insensitive cells.

Our patient had a marked reduction in his massive tumor burden following hormonal therapy alone for six months, far greater than the reported average reduction noted in benign and malignant prostate glands. The reduction of PSA from an initial value of over 4,000 to $0.4 \mathrm{ng} / \mathrm{mL}$ is at first surprising, yet consistent with his tumor burden reduction and prior reports. We have for the moment rendered our patient symptom-free and achieved local control of a massive prostate cancer, adding to the ever increasingly complex topic of prostate cancer.

\author{
Thomas A Rozanski. M.D \\ Section of L 'rology \\ The University of Michigan \\ Medical Center \\ Ann Arbor. Michigan 48109-0.330
}

REFERENCES

1. MacFarlane MT, Abi-Aad A, Stein A, Danella J, Belldegrun $A$, and DeKernion JB: Nenadjuvant hormonal deprivation in patients with locally advanced prostate cancer J Urol 150: $132-134,1993$

2. Oesterling JE, Andrews PE, Suman VI, Zincke H, and Myers RP: Preoperative androgen deprivation therapy: artificial lowering of serum prostate specific antigen without downstaging the tumor. J Urol 149: 779-782. 1993.

3. Kennedy TJ, Sonneland AM, Marlett MM, and Troup RH: Luteinizing hormone-releasing hormone downstaging of clinical stage C prostate cancer. J Urol (Pt. 2) 147: 891-893. 1992.

4. Eri LM, and Tveter KJ: A prospective, placebo-controlled study of the luteinizing hormone-releasing hormone agonist leuprolide as treatment for patients with benign prostatic hyperplasia. J Urol 150: 359-364, 1993 